\title{
QUALITATIVE PROCESS AUTOMATION
}

Maj Steven R. LeClair and Ms Frances L. Abrams

Air Force Wright Aeronautical Laboratories, Materials Laboratory

Wright-Patterson Air Force Base, Ohio

\section{ABSTRACT}

This paper addresses basic research in computer science, control theory and material processing science wherein the focus is to develop a process control system which enables in-situ process model development. A generic control system architecture employing the use of Qualitative Physics (QP) is described together with an explanation of the system operation involving multiple cooperating expert

systems. Specific details involving development and application of the control system to autoclave curing of composites is also be presented together with recent research results.

\section{INTRODUCTION}

Qualitative Process Automation (OPA) is more than a qualitative approach to process control. It is a control philosophy which advocates on-line 'control-cycle' generation. The fundamental research is sue is the formalization of a canonical representation for process. Such a formalization would enable a control system to independently interpret heterogeneous sensor data, and qualitatively reason about that data in order to construct a processing plan in real-time. In theory, these systems will know better how to process a material than we humans do because they will possess the necessary material and processing knowledge to adapt in-situ and self-improve over time. The long-term goa 1 of this research is to enable the deployment of intelligent process control systems across both the defense and commercial sectors to improve both quality and yield in discrete part manufacturing.

The genesis for QPA began in January 1986 with a joint research program to address the limitations in processing graphite (fiber) - epoxy (resin matrix) composite materials. The program involved the teaming of two research groups - one focused on advanced composites and the other on manufacturing. The objective of the research was to extend existing control theory through the use of 'qualitative physics' [1] to enable a material processing system to generate a process control strategy on-line (during the process) and therefore, specifically tailor the process to the conditions of the material and process history.

The QPA architecture was developed by making use of, not only qualitative physics, but concurrent expert systems cooperating together through a 'blackboard' communication system [2]. The QPA system uses 'process-specific' knowledge coupled with expert heuristics to interpret multiple, heterogeneous sensed data and represent the data in symbolic (or qualitative) form. At regular (every 30 seconds for the implemented system) intervals throughout the process this symbolic information is updated and compared with 'expected' process states. Control heuristics are then used to perform temporal reasoning about past and present process states for the purpose of predicting a future state. Control is exercised by issuing commands to adapt the process agent (autoclave) for desired results based on periodic future state predictions.
In addition to enabling 'dynamic control' of nonlinear process response, qualitative techniques provide for the development of process control systems which are flexible to change, i.e.. the evolution of processing knowledge through both external (human) intervention and system selfimprovement. The parsing of symbolic sensor data enhances the ability of material processing systems to consider numerous combinations of parameters within imposed time constraints. Finally, such systems are also capable of managing multiple (potentially conflicting) process goals.

A prototype QPA system for autoclave cure of graphite-epoxy composites has been demonstrated to successfully control the autoclave process for making advanced composite materials for a number of materials and part geometries. In so doing, the properties of QPA generated parts equal or surpass the chemica7, physical and mechanical properties obtained using conventional techniques (Figure 1 ). In addition, QPA has demonstrated dramatic improvements in material processing times as a result of $i$ ts ability to adapt on-line (i.e., in-situ) to material processing behavior and to generate tailored cure cycles (Figure 2). The long term manufacturing objective of the QPA research is to formalize qualitative techniques for use in material processing and to develop a generic process control system architecture which can be applied to a large variety of processes.

\begin{tabular}{|c|c|c|c|}
\hline & QPA & Standard \\
\hline Fiber Volum & & $63-68 \%$ & $59-70 \%$ \\
\hline Void Conten & & $<1 \%$ & $<1 \%$ \\
\hline Dble. Notch & Shear & 10.55 & $10.44 \mathrm{ksj}$ \\
\hline 0 Tension & $\begin{array}{l}\text { Strength } \\
\text { Modulus }\end{array}$ & $\begin{array}{l}\text { 273ksi } \\
21 \mathrm{Msi}\end{array}$ & $\begin{array}{l}\text { 260ksi } \\
21 \mathrm{Msi}\end{array}$ \\
\hline 90 Tension & $\begin{array}{l}\text { Strength } \\
\text { Modulus }\end{array}$ & $\begin{array}{l}8.2 \mathrm{ksi} \\
1.6 \mathrm{Msi}\end{array}$ & $\begin{array}{l}7.5 \mathrm{ksi} \\
1.4 \mathrm{Msi}\end{array}$ \\
\hline Degree of $\mathrm{C}$ & ure & $98-100 \%$ & $>98 \%$ \\
\hline
\end{tabular}

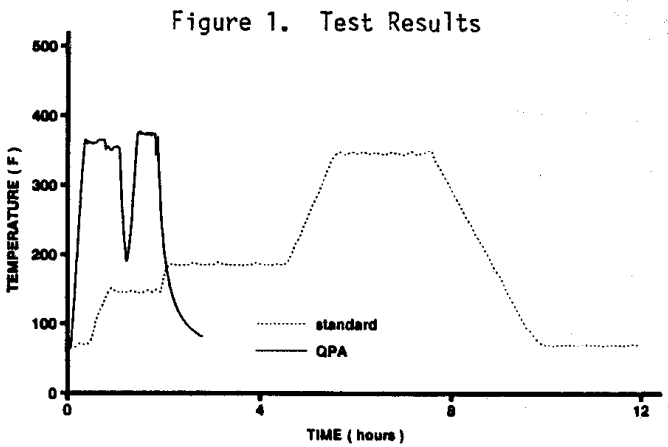

Figure 2. Comparison Between QPA and Standard Cure Cycles 


\section{SCOPE}

The creation of the term Qualitative Process Automation is intended to focus attention on those characteristics which distinguish QPA from traditional process control strategies. The system uses qualitative (symbolic) expressions for relating control outputs to process behavior rather than the quantitative algorithms which characterize traditional controller systems. Quantitative sensor data is converted into symbolic data, which imparts more meaning about the state of the process. The goals of the process are also represented symbolically and are used to make control decisions based on material properties which determine the end quality of the part.

QPA control, and therefore the process strategy. is focused on the quality of the product rather than on the behavior of the processing vessel as is the case with control systems using a predetermined cycle. Sensor data, directly related to product quality, is used to determine progress towards quality goals and to warn of degrading process deviations. The process controls are then manipulated according to heuristics in order to bring part quality into line with goals. while the process is in-progress, and without prior development of a fixed process cycle. (4)

Finally, the word 'automation' implies much more than mere process control. The combined effect of qualitatively representing process and material specific information is a system which has adequate knowledge to arrive at it's own control cycle (decision path from initial state to goal state) and is thus capable of automating the process. Conventional process cycles for batch materials processing are usually developed off-line by experts. The most advanced experts use process models as tools for process cycle development. The cycles are procedurat, and although developed with the best and most current knowledge available they are based on average material parameters and process conditions. The resulting 'recipes' are usually proven using costly trial and error process runs, and when conditions fall outside the specified conditions, products are rejected. The QPA system automates, not on $7 y$ the control of the process, but the development of the cure cycle. Further, because QPA develops the process procedure adaptively, during real-tine, the processes are developed not for the average conditions but for the specific conditions of each process run.

QPA is much more automated than most expert systems, in that it does not interact with an operator once a process has begun, but rather only with the data it derives from sensors and the controls it adjusts automatically. The qualitative knowledge and heuristics are adequate to develop processes for a variety of materials and part geometries without user input. In the prototype autoclave controller at the Materials Laboratory, the QPA system automatically manipulates temperature and pressure in a laboratory scale autoclave to cure advanced composite parts of a variety of materials and geometries.

Not every process is appropriate for QPA. Steady state operations, especially those with relatively few variables, are often amenable to maintenance by adjustment using traditional quantitative algorithms and, where applicable, quantitative control is more precise. Also, processes where the product quality is directly controllable by manipulation of a single parameter (which can also be directly measured) may be little improved by these methods. Many processes of interest, however, are not steady state, and involve the non-linear control of several parameters, some of which can only be measured indirectly, or inferred from other measurements. The relationship between those parameters which can be directly controlled and the parameters we wish to control may be complex, indirect, and poorly understood. The autoclave curing of advanced composites is only one example of such a process.

Continuous processes can be handled by watching the process for certain process states which are disturbances to the desired state. Batch processes. which are commonly used in the manufacturing of materials, generally go through phases of operation. At the very least there is a transitory startup phase, the main body of the process, and another transitory shutdown phase. During these phases the goals and process relationships may be different. Any batch chemical process, for instance, will usually involve considerable change in response to control input over the course of the process.

The use of heuristics and QP theory in process control has gained increasing popularity in the past few years $(5,6)$. These systems have not been completely closed loop however. Instead, they rely on human operators to act on their advice. Such systems are reliant on operators who are consistently attentive and can reliably understand the advice of a computer controlled system.

\section{THE QPA ARCHITECTURE}

\section{Software}

In order to develop and control a process, the QPA system must accomplish several tasks. It must gather data and interpret that data, first into simple symbolic form, and then into a more complex concept of the state of the process. It must then evaluate this process state, considering the goals of the process, and make control decisions, and transmit control commands to the process vesse 1 in quantitative form.

The OPA knowledge base for the prototype autoclave system is divided along the lines of these responsibilities, then, into three separate knowledge bases. The communication of information between these knowledge bases, with the environment of the controller, is through a blackboard or common memory location. This contains both quantitative and symbolic information about the past and present sensor data and process state.

Communication with the environment takes the form of obtaining sensor data through interface(s) such as $A / D$ devices and serial ports, posting data and process state information to CRTs for user viewing, obtaining keyboard inputs from the user, archiving data to files, and outputting control signals to the process agent (reaction vessel. autoclave, press, etc.). Communication between the expert systems involves the posting of data and decisions resulting from each and flagging the information as ready for processing by the others. The expert system which performs these functions in $Q P A$ is called the Blackboard (BB) Monitor.

In order to use data for heuristic decision making, it must be converted to symbolic form. There are two levels of symbolic information developed by the second expert system of QPA. The first level pertains to individual sensors. Quantitative sensor data by itself means little in symbolic terms. In order to be of value it must first be compared with other values and then a symbol assigned to describe 
that comparison. Temperatures, for instance, may be compared with thermal limits on the processing equipment or material, or with past temperatures in the same location, to give second order data with respect to time. (7)

Sensor data from a single source is usually incomplete, and can be subject to error and sensor failure. A real-time control system then must fuse the data from several sources to give a more complete and reliable picture of the process state. QPA makes use of multiple sensors and the second level of symbolic information relates the data from different sensors to patterns of process behavior. This parsing of data on two levels is essentia? in determining a process state and direction on which decision making can be based. The function is accomplished by the QPA system with a second expert system called the Parser.

Finally, the Thinker expert system system uses the results of parsing and the historical information of past decisions to verify the parser results and compare them to the process goals. It then decides, based on heuristics and a set of qualitative 'influences', the appropriate control adjustment required for responding to the current state of the process. If there are conflicting control requirements, it uses conflict resolution rules to select the best control responses and issues them as commands to the BB monitor.

Process states are represented in the knowledge bases of both parser and thinker as object oriented frames. There are two types of frames. The first type is called a process episode. Frames of this type allow the QPA system the capability to respond to changes in process goals and response to controls, with time, if the process is a batch operation and there is a clear and necessary sequence of events. These frames contain lists, or agendas, of the second type of frame, process instances. These highlight important control concerns during that episode. Process goals are, in a sense, subdivided into episode goals, which are listed as transition process instances.

Process instance frames are activated, not in sequence, but whenever the preconditions required for the particular frame to be active have been met. These preconditions are represented as rules to the parser. However, the thinker will not act on an active process instance unless it is on the agenda of the current episode. If it is on the agenda, the frame includes instructions on what to do when it becomes active. Sometimes the means to affect the process instance is a direct change to one of the controllable parameters. Sometimes however, the thinker must chain through a set of influence rules to determine the controllable which is most appropriate to produce the desired alteration of process state.

The current version of QPA makes use of a shell called Expert 5, written in the FORTH language for the PC. FORTH was chosen for both compactness and speed. The shell was tailored to the requirements of real-time process control by incorporation of the agenda style of processing and by providing support for blackboard operations as well as operation on multiple co-processors. (8)

\section{Hardware}

The hardware configuration of the QPA system is shown, with autoclave connections, in Figure 3 . The system uses a separate microprocessor for each expert system. This allows parsing and thinking to be done simultaneously. Separate processing of thinking and parsing may not be necessary for all control systems if time demands are not critical. It is clearly advantageous to have a separate processor for the data handling work of the $\mathrm{BB}$ monitor. Data handing and communication are the most demanding of time.

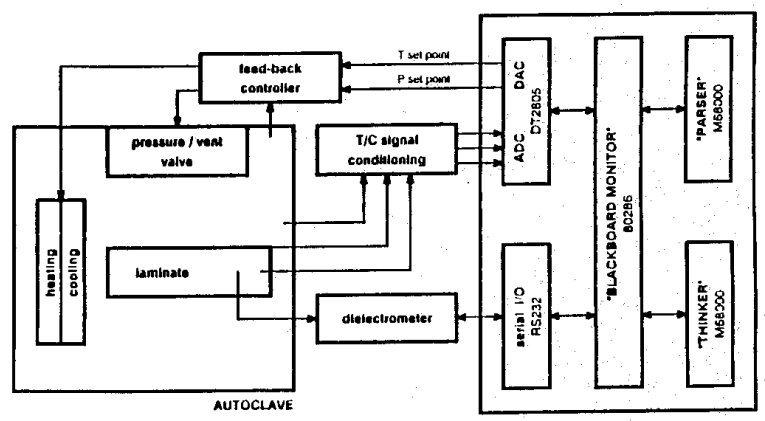

Figure 3. Autoclave with Expert System Control

The data interface is not constrained to the type of sensors shown for the autoclave. Cominercial interface devices are utilized and QPA is written to make communications with the environment straightforward. The entire system is contained in an ATtype personal computer and has been run on several 8086 and 80286 based systems. The co-processor boards are also commercially available. This is a clear advantage for a closed loop controller. The use of small computers lowers both initial and maintenance costs, and allows for dedicated controllers in job shop environments. Further, microcomputers are available as industrially hardened versions, eliminating the need for special housing and wiring. Operators are more likely to be familiar with the personal computer environment, reducing training costs and the discomfort of adaptation.

\section{QPA AS APPLIED TO AUTOCLAVE CURING OF COMPOSITES}

\section{The Autoclave Process}

The example application chosen for QPA was the autoclave curing of composite materials. These strong-lightweight materials are used in many aircraft applications. In the autoclave process, layers of fiber (glass, graphite, polymeric, etc.) which have been previous ly impregnated with a thermosetting polymer resin, are enclosed in a vacuum bag and placed in a large pressure vessel, or autoclave. Here heat is applied to lower resin viscosity and cause a chemical reaction which polywerizes the resin and makes it a solid matrix for transferring load between fibers. While the resin is flowing, pressure is also applied to consolidate or compact the part. The chemical reaction produces its own heat and is called a cure. The original layers are referred to as prepreg and the resulting solid material is referred to as a laminate. Because the Taminate is generally formed into the net shape which is desired for the end use, it may also be referred to as a part.

Only the temperature and pressure of the autoclave and the vacuum within the bag can be controlled. Parameters which need to be controlled such as degree of cure, the degree of compaction, (both of which are difficult to measure) and the result of the interaction of a number of variables must be inferred froin controlled variables. Also, 
the material properties vary with time so the response of the material to control actuation is non-linear with time. In short, the process is an excellent example of the kind of control challenge amenable to the QPA approach. A diagram of the relationships between material properties and process control parameters is shown in Figure 4.

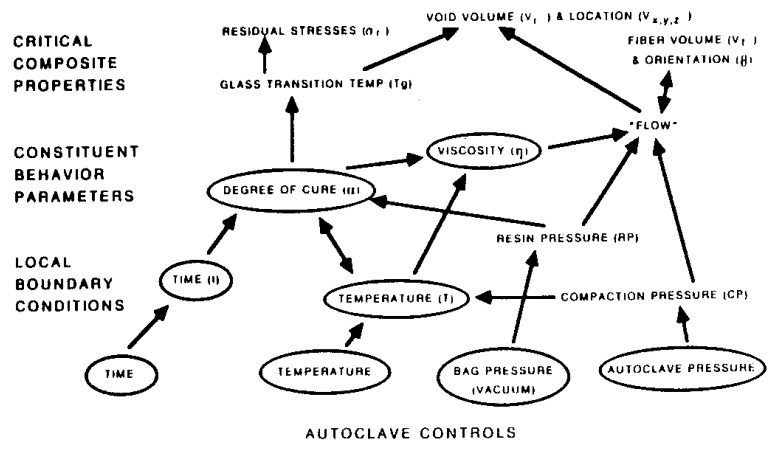

Figure 4. Control and Process Relationships

Aside from the above factors, autoclave curing of composites actually lends itself to a qualitative approach because it is not an extremely rapid process. An average cycle is about $6-8$ hours and real-time response could be as long as several minutes. Also, a long history in research and development of composite processing at the Air Force Materials Laboratory enabled an abundant supply of resources in terms of equipment and expertise.

The temperature and pressure profile (called the cure cycle) for autoclave processing has historically been determined from a combination of experience and trial and error. The trial and error approach is inefficient. To improve on the efficiency of this process, process models have been developed to aid in off-line development of cure cycles. Computer models represent the process under consideration with mathematical equations which are then solved numerically on a computer. It is important to realize that the mathematical equations do not yield the exact solution to the problem. By their nature, the modeling solutions are approximate.

Simple models for predicting the heat transfer and flow of resin through the curing of composite materials are now available. Similar models are being developed for void and residual stress predictions, but models for curing composite materials are invariably incomplete. As with most, if not all material processes, it is virtually impossible to completely describe the process mathematically and to obtain (through measurement) a comprehensive set of the required properties associated with processing hardware and materials. Even if a perfect model could be developed for one set of conditions, process variability due to both the input material and process setup would limit the model's extension to other cases. This same variablility is the prinary reason that pre-established rigid cure cycles fail to reliably produce quality parts.

Further, these models are shortcuts to the same trial and error process as is used for development at the present time. They are capable of predicting. from boundary conditions imposed on the part, what quality will be produced, but not of inferring, from the desired part quality, the control parameters that will provide the required material characteristics.

\section{Control Strategy}

The basic control strategy used in the autoclave application of QPA was to compact and cure the laminate as rapidly as possible without allowing undesirable damage to occur. Compaction gives the material the desired fiber volume fraction and part dimensions, while cure imparts the proper rigidity and temperature resistance to the matrix. Since heat is required for both compaction and cure, and pressure is required for compaction, the goals can be met by heating until cure is done and applying pressure whenever flow allows for compaction. Once cure has occurred, compaction is no longer possible. Fortunately, there is sufficient time at relatively low resin viscosities to complete compaction so the goals may be accomplished more or less simultaneous $7 y$.

It is not always possible to concentrate on ly on positive goals. Damage to the part or the processing vessel can occur by several mechanisms. One is the obvious problem of exceeding the temperature or pressure limitations of either. Another is the build-up of residual stresses or unevenness of compaction that can result from large differences in temperature across the part. Voids can result if air is not removed with vacuum prior to flow, or pressure is not applied to the resin to suppress volatile components at elevated temperatures. Finally, in thick laminates, the heat generated by the exothernic cure reaction may build up until the part is destroyed by overheating.

The autoclave cure is a batch process which requires a transitory startup episode (called PRECURE in the current QPA environment) for heating of the matrix resin to start flow and chemical reaction. Once flow, and/or, the chemical reaction, or cure, have begun, the process enters the CURE episode and significant deviations from PRE-CURE behavior may be expected (i.e., heat given off by exothermic reaction may heat the laminate at rates out of proportion to the heat being provided by the autoclave). Finally, the cured laminate, by now a solid, must be cooled to a temperature where it can safely be removed from the autoclave (COOLDOWN episode).

The agendas of PRE-CURE and COOLDOWN are very linited since little is expected to happen during those episodes. In PRE-CURE, bag vacuum is applied to remove air from the prepreg and heat is applied to transition into CURE. The autoclave may reach its maximum setpoint limit during this episode, even though the part will not, so the OVERTEMP process instance is on the agenda and the autoclave is commanded to hold when the OVERTEMP instance becomes active. COOLDOWN has a default action of temperature decrease and pressure hold, both of which are winners in any conflict resolution problem. It was thought that large gradients might be a problem in COOLDOWN, but that has not turned out to be the case. The only item on the agenda is the ENDCURE process instance which automatically shuts down the process.

The CURE episode is the most action oriented, as expected. As in PRE-CURE, the beginning (default) actions are to have pressure off, temperature increasing and bag vacuum on. Once compaction is active, the pressure will be turned on. Damaging process instances, such as an exotherm or a large temperature gradient, lead to control commands which will cause temperature to decrease rather than increase. Usually OVERTEMP has already put the autoclave on hold when the episode starts. Conditions which might lead to voids lead to control commands which cause the venting of the vacuum. 


\section{Results}

The QPA system has been successfully used to cure a number of materials and part geometries. Comparison of the autoclave temperatures for thick and thin laminates and for two different materials are shown in Figures 5 and 6 . In all cases the manufacturers' suggested cure cycles for these materials would have been the same. In the case of the aged 1034C (256 p.ly) laminate, materials made by recommended methods had serious flaws. With the adaptive processes created by the QPA system, not only were these flaws elininated but panels were processed in substantially reduced times. The knowledge base was not altered to account for differences in material behavior, but adapted to the conditions that were measured in-situ.

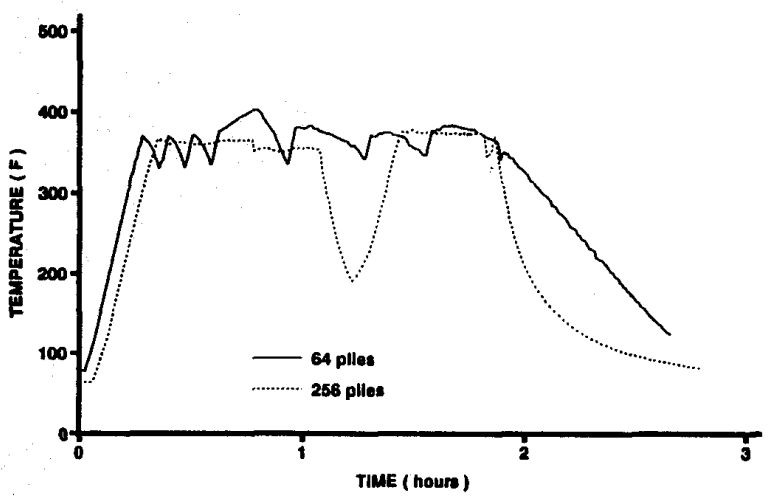

Figure 5. Comparison of Thick and Thin Laminates Cure Cycles

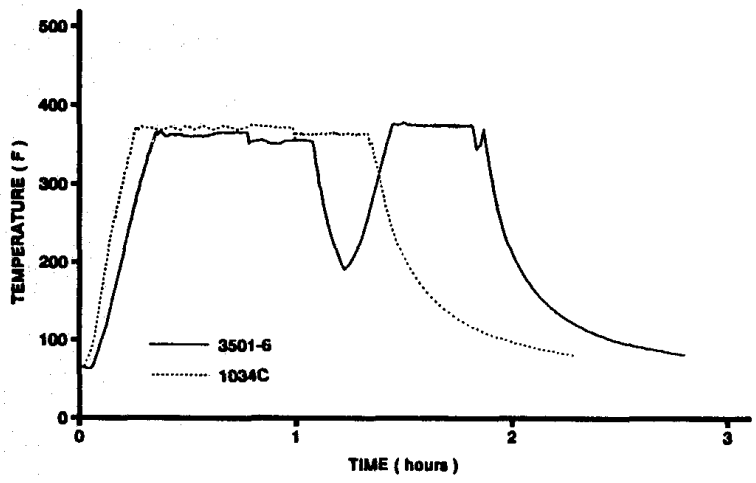

Figure 6. Cure Cycle Comparison 3501-6 and Aged $1034 \mathrm{C}$

\section{CONCLUSIONS}

The autoclave trials indicate that the QPA philosophy, and the current system implementation of that philosophy, offer considerable promise. In composite processing, QPA clearly has the potential of widening the range of acceptable process preconditions without degrading product quality in processes where multiple variables interact to influence the final results. In a world market where rapid transition of ideas to high quality manufactured products is at a premium. OPA offers the advantage of rapid process development as opposed to trial and error methods.

At the current time, the system lacks a number of features which will be necessary in future systems. The sensors are subject to error and failure in any real process. In the autoclave application, error analysis and control was instituted (7) but better methods for verifying sensor integrity and managing the uncertainty of ineasurements are required and studies are underway.

Additional work on knowledge representation is obviously required. The current system uses only relatively short-time trends in the decision making process and does not develop expectations for future process trends. It is clearly advantageous to be able to use both short and long-term trends for description of some process patterns. Expectations would allow for the prevention of some process instances before they occur. Insight into the future would also allow for better handling of the unpredictable time delays for process response to commands. The current software could be modified to include these attributes, but it is possible that another means of representation, either alone, or in combination would handle them better.

At the present time, interaction with the operator, especiality once a run has begun, is extremely limited. Although the system generally operates without any need for operator intervention, in any real environment for process control there. will be situations that require operator attention (e.g. , equipment failure). An industrial system will require the capability of checking its own performance and that of its attached devices, diagnosing faults and allowing operator interrupt without losing track of where it is in the process or allowing dangerous conditions to evolve.

There are other details, specific to the autoclave application which could be improved. Suffice it to say that in any complex process there will be domain-specific knowledge which can be improved. The current approach to that problem is to make the interface for modification of the knowledge base easier to learn and use. In the long range, a self-modifying system with Tearning capabilities and menory is being investigated.

\section{REFERENCES}

1. Forbus, K., "Qualitative Process Theory", Artificial Intelligence, 24, 1984.

2. Abrams, F.L., Lagnese, T.L., LeClair, S.R., \& Park, J.B., Qualitative Process Automation for Autoclave Curing of Composites, Air Force Wright Aeronautical Laboratories Technical Report 37-4083. November 1987

3. LeClair, S.R., "Sensor Fusion: The Application of Artificial Intelligence to Process Control". 1986 Rochester FORTH Conference Proceedings, Rochester, NY, June, 1986, pp 15-22.

4. Abrams, F.L., "An Expert Systen Process Controller for Advanced Composites". Proceedings: Detroit ReTec '87. Society of Plastics Engineers, Detroit. MI, November 2-4 1987.

5. D'Ambrosio, B., Fehling, M.R., Forrest, S., Raulefs, P., Wilber, B.M., "Rea1-Time Process Management for Materials Composition in Chemical Manufacturing", IEEE Expert, 1987.

6. Hajicek, J.D., "A Plastic Injection Molding Expert System", Proceedings: Detroit ReTec '87. Society of Plastics Engineers, Detroit. MI, November 2-4 1987. 
7. Garrett, P., Lee, C.W., \& LeClair, S.R.,

"Qualitative Process Automation vs. Quantitative

Process Control". American Control Conference,

Minneapolis, MN, June 1987.

8. Park, J., "Toward the Development of a Real-Time Expert System", 1986 Rochester FORTH Conference

Proceedings, Rochester, NY, June 1986, pp 23-33.

ACKNOWLEDGEMENTS

This research was performed at the Materials Laboratory, Wright-Patterson Air Force Base, Ohio as part of a Manufacturing Research Program sponsored

by the Directorate of Electronic and Material

Sciences of the Air Force Office of Scientific

Research, Bolling Air Force Base, Washington, D.C.

The genesis for QPA began in January 1986 with a joint-research program to address the limitations in processing graphite (fiber) - epoxy (resin

matrix) composite materials. The program involved

the teaming of two research groups - one focused on Advanced Composites and the other on Manufacturing,

plus researchers from the University of Cincinnati,

University of Dayton Research Institute, and Mr Jack

Park, an independent scientist. 